\title{
Actual and potential infinity
}

\begin{abstract}
The notion of potential infinity dominated in mathematical thinking about infinity from Aristotle until Cantor. The coherence and philosophical importance of the notion are defended. Particular attention is paid to the question of whether potential infinity is compatible with classical logic or requires a weaker logic, perhaps intuitionistic.
\end{abstract}

\section{Introduction}

Beginning with Aristotle, and until the nineteenth century, the vast majority of major philosophers and mathematicians rejected the notion of the actual infinite. They argued that the only sensible notion is that of potential infinity - at least for scientific or, later, non-theological purposes. In Physics 3.6 (206a27-29), Aristotle wrote, "For generally the infinite is as follows: there is always another and another to be taken. And the thing taken will always be finite, but always different" (2o6a2729). As Richard Sorabji [51] (322-3) puts it, for Aristotle, "infinity is an extended finitude" (see also [26], [27]).

This orientation towards the infinite was endorsed by mainstream mathematicians as late as Gauss [16], who wrote: "I protest against the use of infinite magnitude as something completed, which is never permissible in mathematics. Infinity is merely a way of speaking".

The definitive change in mathematicians' orientation towards the infinite took place only in the late nineteenth century, resulting in large part from pioneering work by Georg Cantor, who showed us how to make mathematical sense of completed infinite collections or sets, and how to assign a size or cardinal number to such sets. Indeed, Cantor's theory of infinite sets and numbers proved so elegant, insightful, and useful for 
mathematical purposes that it was quickly assimilated into mathematical practice, where it came to serve an important foundational role. Of course, the set theoretic paradoxes discovered around the turn of the century caused alarm. But many mathematicians no doubt agreed with David Hilbert's conviction that a solution can be found and accordingly that "[n]o one shall drive us out of the paradise which Cantor created for us" ([22], 191). Thankfully, in the course of the first half of the twentieth century, our place in Cantor's paradise was secured, not in the way Hilbert envisaged, but thanks to the now-standard axiomatization ZFC and the closely connected iterative conception of sets.

From this point on, the only sustained opposition to the Cantorian conception of the actual infinite came from intuitionists and constructive mathematics. If the existence of a set requires an explicit specification or construction by us, as this alternative tradition maintains, there can be no room for the actual or completed infinite. We can, with some idealization, be said to be able to construct arbitrarily large finite sets. But as finite creatures, it is out of the question that we ever complete the construction of an infinite set. It follows that the only permissible notion of infinite is the potential one.

The aim of this article is to advance our understanding of the notion of potential infinity that has been eclipsed by the Cantorian notion of actual infinity. Let us be absolutely clear: we no more wish to be expelled from Cantor's paradise than did Hilbert. Rather, our goal is to understand the alternative conception of the infinite that dominated until Cantor, both in order to make sense of two millenia of deep mathematical and philosophical thinking, but also in order to examine whether there is anything for post-Cantorian thinkers to learn from this earlier tradition. We believe there is. ${ }^{1}$

We will be particularly interested in some logical questions concerning potential infinity. Inspired, perhaps, by its only recent defender, many philosophers and logicians believe there is a connection between potential infinity and intuitionistic logic. Others deny the existence of any such connection. After all, thinkers from Aristotle until Gauss rejected the actual infinite in favor of the potential, but never questioned the law of excluded middle.

\footnotetext{
${ }^{1}$ The notion of potential infinity is still with us, perhaps in a more subtle form. It is now a commonly held view in linguistics that languages are infinite. Noam Chomsky [9], for example, once wrote that a grammar projects from a finite corpus to a "set (presumably infinite) of grammatical sentences" (p. 15). It is, we think, more natural to think of a language as potentially infinite. For another example, one of us once asked a teacher about the infinite tape of each Turing machine. He was told that we do not have to think of the tape as (actually) infinite. It is enough to live near a tape factory.
} 
This current state of uncertainty and confusion concerning potential infinity is manifested in some questions raised by the acclaimed logician and philosopher William Tait.

Both Hilbert and the early intuitionists have associated commitment to the actual infinite with the use of classical logic, so that, for example, the use of quantification over the integers combined with classical logic commits one to the set of integers as an actual infinity. I would like someone to explain why this is the same notion of actual infinity as Aristotle's. (One might ask, too, whether quantification over the integers using intuitionistic logic commits one to the actual infinite - and why. $)^{2}$

We thus have the following questions:

(A) If the natural numbers are merely potentially infinite, are we entitled to quantify over all of them using (at least) intuitionistic logic?

(B) Does quantification over all the natural numbers with classical logic presuppose actual infinity?

These questions ask about connections between potential infinity and intuitionistic logic. We must distinguish between a direct connection between the two ideas and a "common cause". It is hardly news that some thinkers postulate a common cause, namely the anti-realism that forms the heart of the usual intuitionistic philosophy. According to this antirealism, the only legitimate sense in which a mathematical statement can be true is by being proved. This anti-realism would support both intuitionistic logic (in ways that have been extensively examined in the philosophical literature) and a rejection of actual infinity (in the way sketched above).

It would be far more interesting and surprising if we could find a direct connection between potential infinity and intuitionistic logic. Michael Dummett claims that there is such a connection and that this provides a new argument for intuitionistic logic, which is independent of the more familiar arguments that proceed via the mentioned antirealism. ${ }^{3}$ His contention is that quantification over a domain that is "indefinitely extensible" - as for example any potentially infinite domain

\footnotetext{
${ }^{2}$ See http://www.cs.nyu.edu/pipermail/fom/2015-March/018602.html. A good follow-up question was posed by Fred Richman: Why "should commitment to actual infinity entail classical logic? A number of people seem to think that it does." (http://www.cs.nyu.edu/pipermail/fom/2015-March/018606.html). We have little to say on that question.

${ }^{3}$ See [12], ch. 24. A similar argument can be found in the second half of [11].
} 
would be-must be intuitionistic. Unfortunately, the contention has never been substantiated. But if correct, it would be significant. Although any potential infinity is indefinitely extensible, the notion of indefinite extensibility is more general. Even if we side with Cantor, against Aristotle, on the nature of the infinity exemplified by the natural numbers, there is a long tradition - which occasionally surfaces even in Cantor's thinking - of regarding the notion of a set as indefinitely extensible (see below).

We proceed as follows. In the next section, we take a closer look at the notion of potential infinity that figured in the mathematical and philosophical tradition. Then, we use the resources of modal logic to provide an analysis of potential infinity. The later sections develop this analysis and explore its consequences. This yields answers to all of the questions raised above. Our analysis shows that potential infinity is not inextricably tied to intuitionistic logic. There is an interesting explication of potential infinity that sanctions classical logic, while still differing in important ways from actual infinity. However, we show that a stricter explication of potential infinity does lead to intuitionistic logic. We emphasize that this route to intuitionistic logic flows directly from our strict explication of potential infinity and does not depend on antirealist premises. This shows that the connection between intuitionistic logic and philosophical anti-realism is less tight than is often assumed. ${ }^{4}$ We take this clarification of the relation between potential infinity, intuitionistic logic, and philosophical anti-realism to be one of the main achievements of the paper.

\section{A brief history of potential infinity}

Aristotle, along with ancient, medieval, and early modern mathematicians, recognized the existence of certain procedures that can be iterated indefinitely, without limit. Examples are the bisection and the extension of line segments. Ancient mathematicians made brilliant use of such procedures. For example, the method of exhaustion, a kind of forerunner to integration, was employed to calculate the areas of curved figures in terms of rectilinear ones.

What was rejected are what would be the end results of applying these procedures infinitely often: self-standing points, infinitely long lines and regions, and infinite sets. The philosophers and mathematicians would also object to thinking of a sequence as itself an actually infinite entity - but perhaps it is too much of an anachronism to put the

\footnotetext{
${ }^{4}$ Of course, we do not deny the converse connection. Anti-realism can serve as a "common cause" of both potential infinity and intuitionistic logic.
} 
matter in those terms.

In On generation and corruption, Aristotle writes:

For, since no point is contiguous to another point, magnitudes are divisible through and through in one sense, and yet not in another. When ... it is admitted that a magnitude is divisible through and through, it is thought that there is a point not only anywhere, but also everywhere in it: hence it follows that the magnitude must be divided away into nothing. For there is a point everywhere within it, so that it consists either of contacts or of points. But it is only in one sense that the magnitude is divisible through and through, viz. in so far as there is one point anywhere within in and all its points are everywhere within it if you take them singly. (317a3-8)

Closely related to the notion of potential infinity here is that of potential existence. For Aristotle, the points interior to a line segment only exist potentially. They are places where the line can be broken. But if the line is not broken at a given place, then the point only exists potentially. ${ }^{5}$ The same goes for the parts of the line segment themselves. As a continuous magnitude, the line segment is a unity. Its parts exist only potentially.

Jonathan Lear [26] argues that it is not the existence of iterated procedures that makes for Aristotelian potential infinity. The matter concerns the structure of geometric magnitudes:

... it is easy to be misled into thinking that, for Aristotle, a length is said to be potentially infinite because there could be a process of division that continued without end. Then it is natural to be confused as to why such a process would not also show the line to be actually infinite by division. ... [I]t would be more accurate to say that, for Aristotle, it is because the length is potentially infinite that there could be such a process. More accurate, but still not true ... Strictly speaking there could not be such a process, but the reason why there could not be is independent of the structure of the magnitude: however earnest a divider I may be, I am also mortal. ... even at that sad moment when the process of division does terminate, there will remain divisions which could have been made. The length is potentially infinite not

${ }^{5}$ This is the central feature of Aristotle's resoluion to some of Zeno's paradoxes. 
because of the existence of any process, but because of the structure of the magnitude. (p. 193)

According to Lear, then, a line segment is potentially infinite because there are infinitely many places where it can be divided. So, no matter how many times one divides a line, there will still be some of the line left. Lear concludes that Aristotle's thesis is "that the structure of the magnitude is such that any division will have to be only a partial realization of its infinite divisibility: there will have to be possible divisions that remain unactualized" (p. 194). Notice, however, that on either Lear's reading of Aristotle or the above conception in terms of iterated procedures, potential infinity invokes both modality and the activities of a perhaps idealized mathematician. ${ }^{6}$

There is a closely related matter. It is generally agreed that Euclid's Elements captures at least the spirit of geometry during Plato's and Aristotle's period. Most of the language in the Elements is dynamic, talking about what a (presumably idealized) geometer can do. For example, the First Postulate is "To draw a straight line from any point to any point", and the Second is "To produce a finite straight line continuously in a straight line". Or consider the infamous Fifth:

That, if a straight line falling on two straight lines make the interior angles on the same side less than two right angles, the two straight lines, if produced indefinitely, meet on that side on which are the angles less than the two right angles.

Book 10, Proposition 1, is an Archimedean principle, also cast in dynamic terms:

Two unequal magnitudes being set out, if from the greater there be subtracted a magnitude greater than its half, and from that which is left a magnitude greater than its half, and if this process is repeated continually, there will be left some magnitude less than the lesser magnitude set out.

Plato was critical of the geometers of his day, arguing that this dynamic language is inconsistent with the nature of the true subject matter

\footnotetext{
${ }^{6}$ We make no attempt here (or anywhere else) to be faithful to everything, or even most things, that Aristotle himself says about modality. Our plan here is to invoke some technical notions from contemporary modal logic and metaphysics in an attempt to explicate a broadly Aristotelian notion of potential infinity. It has a role to play in at least some philosphy of mathematics.
} 
of geometry: ${ }^{7}$

[The] science [of geometry] is in direct contradiction with the language employed by its adepts ... Their language is most ludicrous, ... for they speak as if they were doing something and as if all their words were directed toward action ... [They talk] of squaring and applying and adding and the like ... whereas in fact the real object of the entire subject is ... knowledge ... of what eternally exists, not of anything that comes to be this or that at some time and ceases to be. (Republic, VII)

This, of course, is a nice Platonic thought - for those who like such thoughts. Aristotle rejected this orientation and, we suggest, the dynamic language employed in Ancient geometry better reflects his views. The matter of infinity is tied to this. For Aristotle, we never have infinite collections of points, objects, or anything else, and we never have infinitely long lines or infinitely large regions of space or time. Because of the structure of the geometric magnitudes (to echo Lear), we have procedures that can be iterated indefinitely, and we speak about what those procedures could produce, or what they will eventually produce if carried sufficiently (but only finitely) far. In holding that these geometric procedures can be iterated indefinitely, Aristotle again follows the mathematical practice of the time, this time in opposition to his other major opponents, the atomists (see [37]), who postulate a limit to, say, bisection.

As noted, when it comes to the infinite, views like Aristotle's were standard throughout the medieval and early modern period, through most of the nineteenth century. The greatest mathematical minds insisted that only the potentially infinite makes sense. Leibniz, for example, wrote:

It could ... well be argued that, since among any ten terms there is a last number, which is also the greatest of those numbers, it follows that among all numbers there is a last number, which is also the greatest of all numbers. But I think that such a number implies a contradiction ... When it is said that there are infinitely many terms, it is not being said

\footnotetext{
${ }^{7}$ According to Proclus [42] (p. 125) the "problem" of dynamic language in geometry occupied those in Plato's Academy for some time. It might be noted that the Euclidean geometry in Hilbert [21] uses a more static language. For example, Hilbert's first axiom is "For every two points $A, B$ there exists a line a that contains each of the points $A, B$." At least that much would be more to Plato's liking.
} 
that there is some specific number of them, but that there are more than any specific number. (Letter to Bernoulli, [28], III 566, translated in [31], 76-77, 87) ${ }^{8}$

... we conclude ... that there is no infinite multitude, from which it will follow that there is not an infinity of things, either. Or [rather] it must be said that an infinity of things is not one whole, or that there is no aggregate of them. ([29], $6.3,503$, translated in [31], 86)

Yet M. Descartes and his followers, in making the world out to be indefinite so that we cannot conceive of any end to it, have said that matter has no limits. They have some reason for replacing the term "infinite" by "indefinite", for there is never an infinite whole in the world, though there are always wholes greater than others ad infinitum. As I have shown elsewhere, the universe cannot be considered to be a whole. $([30], 151)$

We can add the passage from Gauss [16] mentioned at the outset of this article: "I protest against the use of infinite magnitude as something completed, which is never permissible in mathematics. Infinity is merely a way of speaking". ${ }^{9}$

For Gauss, as for Leibniz, as for Aristotle, as for a host of others, the infinite just is the limitlessness of certain processes; no actual infinities exist. The only intelligible notion of infinity is that of potential infinitythe transcendence of any (finite) limit.

At least for the cases of interest here - regions, natural numbers, and the like-Georg Cantor argued for the exact opposite of this, claiming that the potentially infinite is dubious, unless it is somehow backed by an actual infinity:

I cannot ascribe any being to the indefinite, the variable, the improper infinite in whatever form they appear, because they are nothing but either relational concepts or merely subjective representations or intuitions (imaginationes), but never adequate ideas ([6], 205, note 3$)$.

\footnotetext{
${ }^{8}$ The "contradiction" mentioned here might be the so-called "Galileo paradox", that with infinite collections, a proper subset can be equinmerous with a set. This, of course, is now a standard feature of infinite sets, a feature and not a bug.

${ }^{9}$ To be sure, Leibniz, Gauss, and a host of others were not completetely consistent on this. They were, after all, pioneers in the emergence of modern mathematics, not to mention their use of infinitesimals. The exegetical issues are (well) beyond the scope of this paper.
} 
... every potential infinite, if it is to be applicable in a rigorous mathematical way, presupposes an actual infinite ([7], 410-411).

We think it safe to say that this Cantorian orientation is now dominant in the relevant intellectual communities, especially concerning the mathematical domains mentioned above. Notable exceptions are various constructivists, who reject the actually infinite (stay tuned).

It should be noted that, on the surface, at least, Cantor was not consistent in his rejection of the potential infinite. Sometimes he ascribed to the so-called "absolutely infinite", or what he dubbed "inconsistent multitudes" (e.g., the ordinals), features closely analogous to those of the potentially infinite. In a much quoted letter to Dedekind, in 1899, he wrote:

[I]t is necessary ... to distinguish two kinds of multiplicities (by this I always mean definite multiplicities). For a multiplicity can be such that the assumption that all of its elements 'are together' leads to a contradiction, so that it is impossible to conceive of the multiplicity as a unity, as 'one finished thing'. Such multiplicities I call absolutely infinite or inconsistent multiplicities ... If on the other hand the totality of the elements of a multiplicity can be thought of without contradiction as 'being together', so that they can be gathered together into 'one thing', I call it a consistent multiplicity or a 'set'. (Ewald [14], 931-932)

An 1897 letter to Hilbert is even more suggestive:

I say of a set that it can be thought of as finished ... if it is possible without contradiction (as can be done with finite sets) to think of all its elements as existing together ... or (in other words) if it is possible to imagine the set as actually existing with the totality of its elements. (Ewald [14], 927)

Of course, Cantor considered all of the transfinite sets, such as the natural numbers and the real numbers, to be actual infinities. ${ }^{10}$ Our present concern is with those; we plan to address the potentiality of the iterative hierarchy elsewhere (see Linnebo [33]).

At least with hindsight, it is clear what Aristotle, and the ancient, medieval, and early modern mathematicians did not have: infinitely

\footnotetext{
${ }^{10}$ Jané [23] is an insightful discussion of these themes in Cantor's thought at various stages of his career.
} 
large, completed collections and infinitely large geometric figures. But what did they have instead? Just what is a potential infinity?

Many contemporary thinkers are skeptical that a workable notion of potential infinity can be made out which doesn't just collapse to the standard Cantorian notion of actual infinity. A good examplar of this attitude is Karl-George Niebergall [39], who contends that "a clear meaning has never been given to" the phrases " $x$ is potentially infinite" and "T makes an assumption of the potentially infinite" (p. 231). He later expands as follows:

I simply have no ordinary understanding of these phrases, and I do not find much help in the existing literature on them. It seems that even examples are missing ... [T] hose philosophers who are interested in the theme of the potentially infinite are usually drawn to it because they regard it as desirable to avoid assumptions of infinity (i.e., of the actual infinity), yet do not want to be restricted to a mere finitist position. An assumption of merely the potentially infinite seems to be a way out of this quandary: ... it allows you to have your cake and eat it too (p. 256-7).

Niebergall [38], §§2.5,3; [39], §6 argues that, on some straightforward attempts at definition, the potentially infinite just collapses into the actually infinite (or the finite). So, what we cannot have, he claims, is a notion of the potentially infinite that is distinct from both the finite and the actually infinite. If we follow contemporary practice and allow the actually infinite a place, then there is no room for the merely potentially infinite. Everything is either finite or infinite - nothing can fit between those. ${ }^{11}$

Niebergall [39], 257 makes one concession, however: "It is granted that one could try to define ' $x$ is potentially infinite' by employing a modal vocabulary." This is the strategy that we will explore. We hope to articulate a serviceable notion of the potentially infinite, which can live alongside the actually infinite. This plays a role in understanding the notions of mathematical "construction", in indefinite extensibility, and in the debate over absolute generality. It will also help to articulate the thesis that the cumulative hierarchy of sets is itself potential.

\footnotetext{
${ }^{11}$ Niebergall's argument here relies on the law of excluded middle. Charles McCarty [36] shows how to capture the notion of a set that is not finite and also not infinite (on straightforward definitins of those notions) in an intuitionistic background. Even in a classical setting, one can consistently maintain the existence of a set that is not finite and also not Dedekind-infinite, by rejecting the axiom of dependent choice. One of our goals here is to develop a workable notion of potential infinity that is substantially independent of the backgrould logic and set theory.
} 
This strategy requires a substantive use of modal notions in mathematics. Niebergall thus notes that it clashes with the dominant contemporary view that in mathematics, "talk of possibility and necessity becomes dispensable" since "a mathematical sentence is regarded as necessary if true" (p. 258). It is therefore incumbent on us to say something about the modality involved, and to distinguish it from the sense in which every sentence of pure mathematics is "necessary if true".

\section{Modal explications of potential infinity}

Aristotle, we recall, characterizes the infinite as follows: "there is always another and another to be taken. And the thing taken will always be finite, but always different" (Physics, 206a27-29). Aristotle's claim that matter is infinitely divisible provides a nice example. Consider a stick. However many times one has divided the stick, it is always possible to divide it again (or so it is assumed).

It is fairly natural to explicate Aristotle's temporal vocabulary in a modal way. This yields the following analysis of the infinite divisibility of a stick $s$ :

$$
\square \forall x(P x s \rightarrow \diamond \exists y P y x)
$$

where Pxy means that $x$ is a proper part of $y$. If, by contrast, the divisions of the stick formed an actual infinity, the following would hold:

$$
\forall x(P x s \rightarrow \exists y P y x)
$$

According to Aristotle, it is not even possible to complete infinitely many divisions of the stick, that is:

$$
\neg \diamond \forall x(P x s \rightarrow \exists y P y x)
$$

By endorsing both (1) and (3), Aristotle is asserting that the divisions of the stick are merely potentially infinite, or incompletable, as we will also put it.

According to Aristotle, the sequence of natural numbers too is merely potentially infinite. We can represent this view as the conjunction of the following theses:

$$
\begin{aligned}
& \square \forall m \diamond \exists n \operatorname{SuCC}(m, n) \\
& \neg \diamond \forall m \exists n \operatorname{SuCC}(m, n)
\end{aligned}
$$

Thus, provided we are willing to use the resources of modal logic, there is no problem whatsoever in distinguishing the merely potential infinite from the actual infinite. 
Informal glosses aside, the language of contemporary mathematics is strictly non-modal. Thus, when the question of the appropriate logic for reasoning about potential infinity arises, it typically does so in the ordinary, non-modal language of arithmetic. This is the context in which Hilbert, Brouwer, Dummett, and others mentioned above discuss the question. We thus need a translation to serve as a bridge connecting the non-modal language in which mathematics is ordinarily formulated with the modal language in which our analysis of potential infinity is developed. Suppose we adopt a translation $*$ from the non-modal language, say $\mathcal{L}$, to the corresponding modal language, say $\mathcal{L}^{\diamond}$. The question of the right logic of potential infinity is the question of which entailment relations obtain in $\mathcal{L}$. To determine whether $\phi_{1}, \ldots, \phi_{n}$ entail $\psi$, we need to apply the translation and ask whether $\phi_{1}^{*}, \ldots, \phi_{n}^{*}$ entail $\psi^{*}$ in the relevant modal system. This means that the right logic of potential infinity depends on two factors. First, the logic obviously depends on our modal analysis of potential infinity; in particular, on the modal logic that is used in this analysis. Second, the logic also depends on the bridge that we choose to connect the non-modal language of ordinary mathematics with the modal language in which our analysis of potential infinity is given. Both factors will be investigated in what follows.

This approach to the logic of potential infinity opens the door to an interesting combination of logical orthodoxy and heterodoxy. One can be orthodox concerning the logic of the modal system; adopting some form of classical modal logic. Even so, non-classical logical principles may nonetheless end up being validated in the non-modal language via the translation $*$. From this perspective, the single controversial claim that potentialists make is to deny that the non-modal language $\mathcal{L}$ is fully explicit. To make its claims explicit, we need to apply the translation *. Suppose this single controversial claim is right. Then there is no room for further controversy concerning inferential relationships in $\mathcal{L}$. Before such relationships can be determined, the statements in question must always be made explicit. In our case, we explicate by applying the translation $*$.

Before we embark on our investigation of the two identified factors, it will be useful to distinguish some different orientations towards the infinite. Actualism about the infinite unreservedly accepts actual infinities, and thus finds no use for modal notions in mathematics (or at least no use when it comes to the infinity). Actualists maintain that the non-modal language of ordinary mathematics is already fully explicit and thus deny that we need a translation into some modal language. Furthermore, actualists accept classical logic when reasoning about the infinite and typically also accept all of classical mathematics. 
Potentialism is the orientation that stands opposed to actualism. According to this orientation, the objects with which mathematics is concerned are generated successively, and some of these generative processes cannot be completed. There is room for disagreement about which processes can be completed. A traditional Aristotelian form of potentialism takes a very restrictive view, insisting that at any one stage, there are never more than finitely many such objects, but that we always (i.e., necessarily) have the ability to go on and generate more. Generalized forms of potentialism take a more relaxed attitude. Potentialism about set theory provides an extreme example. According to this view, it is impossible to complete the process of forming sets from any objects that are available, but any generative process that is indexed by a set-theoretic ordinal can be completed. ${ }^{12}$ In particular, potentialists about set theory hold that the generation of natural numbers can be completed:

$$
\diamond \forall m \exists n \operatorname{SuCC}(m, n) .
$$

In short, the potentialist about set theory accepts the possibility of the existence of some actual infinities.

Loosely speaking, these different versions of potentialism disagree on the quantitative matter of "the length" of the processes that can be completed. Traditionalists insist that only finite processes can be completed, while generalized forms of potentialism accept that some, but not all, infinite processes can be completed. In this paper, we are mostly concerned with the traditional form of potentialism that denies (6) and accepts only (4).

Potentialists also differ with respect to a qualitative matter. As characterized above, potentialism is the view that the objects with which mathematics is concerned are successively generated and that some of these generative processes cannot be completed. What about the truths of mathematics? Of course, on any form of potentialism, these are modal truths concerned with certain generative processes. But how should these truths be understood?

Liberal potentialists regard the modal truths as unproblematic. In particular, there are modal truths about generative processes in their entirety, including those that cannot be completed. Consider Goldbach's conjecture. As potentialists interpret it, the conjecture says that necessarily any even natural number that is generated can be written as a sum of two primes. Liberal potentialists maintain that this modal question has an unproblematic answer - it is either true or false. Their approach

\footnotetext{
${ }^{12}$ As the quotes from p. 9 reveal, the view can be traced back to Cantor. See Putnam [43] and Hellman [17] for a modal structuralist development of the view, and Parsons [40] and Linnebo [33], for non-structuralist development.
} 
to modal theorizing in mathematics is thus much like a realist approach to modal theorizing in general: there are objective truths about the relevant modal aspects of reality, and this objectivity warrants the use of some classical form of modal logic. In the next few sections, we show that, provided the modal logic is sufficiently strong, liberal potentialists are entitled to classical first-order logic in their ordinary mathematical reasoning about potential infinity.

The matter is closely related to the so-called weak Brouwerian counterexamples to excluded middle. The liberal potentialist insists that there will or will not be a sequence of twelve consecutive 5's somewhere in the decimal expansion of $\pi$. This is not, of course, because the sequence exists all at once, so to speak, as an actual infinity. Rather, the liberal potentialist notes that, in this case, the digits of the sequence are completely determined by a fixed rule, and it is this rule that guarantees that either there will or will not be the run of 5 's. ${ }^{13}$

In later sections, we discuss a view we call strict potentialism, which goes beyond the liberal view by requiring, not only that every object be generated at some stage of a process, but also that every truth be "made true" at some stage. Consider Goldbach's conjecture. If there are counterexamples to the conjecture, then its negation will presumably be "made true" at the stage where the first counterexample is generated. But suppose there are no counterexamples. Given the conjecture's concern with all the natural numbers, it is hard to see how it could be "made true" without completing the generation of natural numbers. This completion would, however, violate the strict potentialists' requirement that any truth be made true at some stage of the process. So how, if at all, can strict potentialists make sense of quantification over all natural numbers? We will solve this problem by providing an account of how a sentence can be "made true" before all the objects with which the sentence is concerned have been generated. The price, as we will see, is the adoption intuitionistic logic.

\footnotetext{
${ }^{13}$ Since our liberal potentialist is a realist (in truth-value) about the modality, we might be tempted to call her a "modal realist", except, of course, that that label has already been taken. None of our characters is a Lewis-style realist about possible worlds.

Ian Rumfitt [46], $\S 7.4$, argues against the intuitionistic use of weak counterexamples (not under that name) against classical logic. If the decimal expansion of $\pi$ does contain the pattern in question, then, of course, that will be discovered eventually. Rumfitt argues that if "the pattern occurs nowhere in the expansion, it will lie in the rule for expaning $\pi$, together with the axioms that characterize the sequence of natural numbers, that it occurs nowhere" (p. 204). Rumfitt thus supports what we call liberal potentialism here, as an interpretation of intuitionism.
} 


\section{The modal logic}

Our next task is to identify the modal logic to be used in our modal explication of potential infinity. ${ }^{14}$ For the time being, we will be neutral on the non-modal part of the logic (and thus neutral on the liberal/potential divide).

It is often useful to invoke the contemporary heuristic of possible worlds, but, again we understand this as only heuristic, as a manner-ofspeaking. Our official theory is formulated in the modal language, with (one or both of) the modal operators as primitive. The modal language is rock bottom, not explained or defined in terms of anything else. To invoke the heuristic, then, the idea is that a "possible world" has access to other possible worlds that contain objects that have been constructed or generated from those in the first world. From the perspective of the earlier world, the "new" objects in the second exist only potentially.

One sort of construction is geometric, following Euclid: the later world may contain, for example, a bisect of a line segment in the first. Or the later world might contain an extension of a line segment from the first world. Other sorts of constructions are arithmetic: the later world might contain more natural numbers than those of the first, say the successor of the largest natural number in the first world. Or, for a third kind of example, the later world may contain a set whose members are all in the first world. Or, to look ahead, beyond the scope of this paper, a given sequence may have one (or more) elements in the later world than it has in the first world.

Our potentialist assumes that every possible world is finite, in the sense that it contains only finitely many objects. That is in line with the rejection of the actually infinite. We make no such assumption here, however. Our goal is to contrast the actually infinite and the potentially infinite, so we need a framework where both can occur (to speak loosely). An actual infinity - or, to be precise, the possibility of an actual infinity - is realized at a possible world if it contains infinitely many objects.

We also assume, without much by way of argument, that objects are not destroyed in the process of construction or generation. This is in keeping with most ordinary mathematical talk about construction. We construct new objects but never destroy old ones. Suppose, for example, that a given line segment is bisected. Then the resulting "world" contains the two bisects, as well as the original line segment.

\footnotetext{
${ }^{14}$ Our approach takes its inspiration from Linnebo [33], which develops a modal explication of the Cantorian notion that the universe of set theory is itself potential (as noted just above).
} 
As noted, we must say something about the modality that we invoke, which motivates a specific modal logic. To continue the heuristic, it follows from the foregoing that the domains of the possible worlds grow along the accessibility relation. So we assume:

$$
w_{1} \leq w_{2} \rightarrow D\left(w_{1}\right) \subseteq D\left(w_{2}\right),
$$

where ' $w_{1} \leq w_{2}$ ' says that $w_{2}$ is accessible from $w_{1}$, and for each world $w$, $D(w)$ is the domain of $w$. For present purposes, we can think of a possible world as determined completely by the mathematical objects - regions, numbers, sets, etc.- it contains. So we can add that if $D\left(w_{1}\right)=D\left(w_{2}\right)$, then $w_{1}=w_{2}$; and we can strengthen the above to a biconditional. However, the above conditional is sufficient for our technical purposes. We will talk neutrally about the extra mathematical objects existing at a world $w_{2}$ but not at an "earlier" world $w_{1}$ which accesses $w_{2}$, as having been "constructed" or "generated".

As is well-known, the above conditional entails that the converse Barcan formula is valid. That is,

$(\mathrm{CBF}) \quad \exists x \diamond \phi(x) \rightarrow \diamond \exists x \phi(x)$

The validity of $(\mathrm{CBF})$ makes it doubtful that the modality in question can be "ordinary" metaphysical modality - whatever exactly that is. For it is widely held that there are objects whose existence is metaphysically contingent (Williamson [56] notwithstanding). For example, let $\phi(x)$ say (or entail) that $x$ does not exist. Presumably, there is someone, such as Aristotle, or a given region, that might not have existed. So we have $\exists x \diamond \neg \phi(x)$. But then it would follow via (CBF) that it is possible for there to exist something that doesn't exist, which is absurd. We are also rejecting the common thesis that the ontology of pure mathematical objects is modally invariant, at least in the metaphysical sense of modality. However, we do have that once a mathematical object comes into existence - by being constructed - it continues to exist, of necessity. In short, we accept generation, but not corruption.

How should the modality used in our explication of potential infinity be interpreted? It is not our aim to provide a definite answer. Our current aim is only to identify structural features of any modality that can be used in the mentioned explication. Let us briefly mention some options, however.

One option is that the modality is a restriction of "ordinary" metaphysical modality. In terms of possible worlds, the relevant modality is the one that results from restricting the accessibility $R$ associated with metaphysical modality by imposing that additional requirement that domains only ever increase along the accessibility relation. More explicitly, 
we define: ${ }^{15}$

$$
w_{1} \leq w_{2}: \leftrightarrow w_{1} R w_{2} \wedge D\left(w_{1}\right) \subseteq D\left(w_{2}\right)
$$

An alternative option is to sever any link between the metaphysical modality and the modality invoked in explicating potential infinity. Instead, we might regard the latter as an altogether distinct kind of modality, say the logico-mathematical modality of [43] or [17], or the interpretational modality of [15] or [33]. As mentioned, we here remain neutral on the exact interpretation of the modal operators, contenting ourselves with identifying some structural features of any plausible interpretation.

Our next issue concerns the right modal logic. Again, it is useful to indulge in talk about possible worlds, writing the associated accessibility relation as $\leq$. Recall that $w \leq w^{\prime}$ means that we can get from $w$ to $w^{\prime}$ by generating more objects. This motivates the following principle:

Partial ordering: The accessibility relation $\leq$ is a partial order. That is, it is reflexive, transitive, and anti-symmetric.

We can also require the accessibility relation to be well-founded, on the grounds that all mathematical construction has to start somewhere. Nothing of substance turns on this, however.

At any given stage in the process of construction, we generally have a choice of which objects to generate. This seems especially relevant in geometry. For example, given two intervals that don't yet have bisections, we can choose to bisect one or the other of them, or perhaps to bisect both simultaneously. Assume we are at a world $w_{0}$ where we can choose to generate objects, in different ways, so as to arrive at either $w_{1}$ or $w_{2}$. It makes sense to require that the licence to generate a mathematical object is never revoked as our domain expands. The option to bisect a given line segment, for example, can always be exercised at a later stage.

This corresponds to a requirement that any two worlds $w_{1}$ and $w_{2}$ accessible from a common world have a common extension $w_{3}$. This is a directedness property known as convergence and formalized as follows:

$$
\forall w_{0} \forall w_{1} \forall w_{2}\left(w_{0} \leq w_{1} \wedge w_{0} \leq w_{2} \rightarrow \exists w_{3}\left(w_{1} \leq w_{3} \wedge w_{2} \leq w_{3}\right)\right)
$$

We therefore adopt the following principle.

\footnotetext{
${ }^{15}$ This restriction can be captured proof-theoretically, using the resources of plural logic (which we explain in Section 7). Let $\phi$ indicate that $\phi$ is metaphysically necessary, and let Exx be a plural existence predicate (either primitive or defined). Then the relevant notion $\square$ of necessity can be defined by letting $\square \phi$ abbreviate $\exists x x(\forall x(x \prec x x) \wedge \boldsymbol{\square}(E x x \rightarrow \phi))$, that is, the metaphysical necessity of $\phi$, conditional on everything that in fact exists still existing.
} 
Convergence: The accessibility relation $\leq$ is convergent.

This principle ensures that, whenever we have a choice of mathematical objects to generate, the order in which we choose to proceed is irrelevant. Whichever object(s) we choose to generate first, the other(s) can always be generated later. Unless $\leq$ is convergent, our choice whether to extend the ontology of $w_{0}$ to that of $w_{1}$ or that of $w_{2}$ might have an enduring effect. ${ }^{16}$

The mentioned properties of the accessibility relation $\leq$ allow us to identify a modal logic appropriate for studying the generation of mathematical objects. Since $\leq$ is reflexive and transitive, the modal logic S4 will be sound with respect our intended system of possible worlds. As is well known, the convergence of $\leq$ ensures the soundness of the following principle as well:

$$
\diamond \square p \rightarrow \square \diamond p .
$$

The modal propositional logic that results from adding this principle to a complete axiomatization of S4 is known as S4.2.

Taking stock, we have completed the first task of identifying the propositional modal logic that governs our modal explication of potential infinity: S4.2. We add to this logic the ordinary rules for the first-order quantifiers. As is well known, this enables us to derive the Converse Barcan Formula:

$$
\text { (CBF) } \quad \exists x \diamond \phi \rightarrow \diamond \exists x \phi .
$$

But that is fine, since we are anyway committed to the validity of this formula. This means that no complications are needed to block the mentioned derivation, such as a free logic or an existence predicate. ${ }^{17}$ The

\footnotetext{
${ }^{16}$ It should be noted that convergence is at least questionable for construction that has some indeterminacy, such as Browerian choice sequences. We plan to treat those in later work. Note also that convergence does not hold for other sorts of modality that relate to producing things. Suppose, for example, that I can either bake bread or bake a cake, since I have enough flour, eggs, water, etc. to do either. So there is one accessible world in which I bake bread and another in which I bake a cake. But if I choose to bake bread, I may no longer be able to bake a cake, as I may no longer have the ingredients. See Barker [4]. This does not present a problem for the present approach, since we restrict attention to cases where the objects added are mathematical in nature, and where there is thus no concern about using up resources.

${ }^{17}$ If one wants to have function symbols, say in order to better reflect ordinary mathematical language, one should adopt a negative free logic. A number $n$ might exist at a given world (representing a stage of the generative procedure) without the successor of $n$ existing there. The details are a distraction here.
} 
question of higher-order extensions of our modal logic will be considered shortly.

The following proves useful in what lies ahead. Say that a formula $\phi$ is stable if the necessitations of the universal closures of the following two conditionals hold:

$$
\phi \rightarrow \square \phi \quad \neg \phi \rightarrow \square \neg \phi
$$

Intuitively, a formula is stable just in case it never "changes its mind", in the sense that, if the formula is true (or false) of certain objects at some world, it remains true (or false) of these objects at all "later" worlds as well.

Are our atomic predications stable? This question becomes very important in the next section. Since our plan is to use the modal framework to develop potentialist and actualist accounts of various kinds of mathematical objects - geometric, arithmetic, analytic, set theoretic - there are many predicates to consider. In all cases, we contend, atomic predications are stable. Whether or not a number is a successor of another won't be affected by any further generation of objects. The same goes for set theoretic membership. A set already generated will neither lose or gain elements as a result of the generation of further sets. For the case of geometry, see [35].

\section{The bridge}

Our next task is to specify a bridge that connects the non-modal language of ordinary mathematics with the modal language in which our explication of potential infinity is given.

The Gödel translation of the language of intuitionistic logic into classical logic provides one well-known bridge. The universal quantifier $\forall$ is translated as $\square \forall$. The existential quantifier translates as itself. (See Appendix A for details.)

We contend, however, that the Gödel translation is inappropriate for our present purposes because it fails to capture the potentialist understanding of the quantifiers. To see this, consider the principle that every number has a successor:

$$
\forall m \exists n \operatorname{SUCC}(m, n)
$$

This is an axiom of both Peano and Heyting arithmetic. But its Gödel translation is

$$
\square \forall m \exists n \operatorname{SUCC}(m, n)
$$


which requires that every world that contains one number, contains all of them. This is precisely what potentialists deny. ${ }^{18}$

At the heart of potentialism lies the idea that the existential quantifier of ordinary non-modal mathematics has an implicit modal aspect. When we say that a number has a successor, we really mean that it potentially has a successor, that is, that it is possible to generate a successor. This suggests that the right translation of $\exists$ is $\diamond \exists$. Since the universal quantifier can hardly be less inclusive in its range than the existential, this also suggests that $\forall$ be translated as $\square \forall$, as it is in the Gödel translation. ${ }^{19}$

So understood, the quantifiers of ordinary non-modal mathematics are understood as devices for generalizing over absolutely all objects, not only the ones available at some stage, but also any that we may go on to generate. In our modal language, these generalizations are effected by the strings $\square \forall$ and $\diamond \exists$. Although these strings are strictly speaking composites of a modal operator and a quantifier proper, we will shortly show that they behave logically just like quantifiers ranging over all entities at all (future) worlds. We will therefore refer to the strings as modalized quantifiers. The proposal is thus that each quantifier of the non-modal language is translated as the corresponding modalized quantifier.

Each connective is translated as itself. Let us call this the potentialist translation, and let $\phi^{\diamond}$ represent the translation of $\phi$. We say that a formula is fully modalized just in case all of its quantifiers are modalized. Clearly, the potentialist translation of any non-modal formula is fully modalized.

\footnotetext{
${ }^{18}$ As noted, it is well known that, when the logic of the modal language is S4, the Gödel translation validates intuitionistic logic. When the modal logic is stronger but still weaker than S5, a logic strictly intermediate between intuitionistic and classical is validated. In particular, when the modal logic is S4.2, as it is here, the Gödel translation validates the sub-classical system that results from of adding to intuitionistic logic so-called 'weak excluded' middle, namely $\neg \phi \vee \neg \neg \phi$. So if you believe the Gödel translation provides the appropriate bridge, you should also believe that the logic of potential infinity is some system strictly intermediate between intuitionistic and classical logic. One might also tinker with the Gödel translation to get it closer to potentialism. For example, one might translate $\exists$ as $\diamond \exists$; or as $\square \diamond \exists$. The result, via the bridge, is a perverse logic that violates uncontroversial quantifier principles such as existential elimination. Another option, perhaps, is to adopt van Dalen's [10] Beth-Kripke semantics, instead of the usual Kripke-style model theory. The details would be a distraction here.

${ }^{19} \mathrm{We}$ would get the same result if we began with the Gödel translation of the universal quantifier and then took the existential quantifier to be the dual of the universal, i.e., if $\exists x \phi$ is defined as $\neg \forall x \neg \phi$. As noted below, however, we are not translating the connectives here.
} 


\section{The first-order logic of potential infinity}

We are now ready to put the pieces together. Recall that we take an entailment to obtain in our non-modal language just in case the corresponding entailment obtains between the translations of the relevant formulas into the modal language in which our analysis of potential infinity is given.

Let us state a key result. Let $\vdash$ be the relation of classical deducibility in a non-modal first-order language $\mathcal{L}$. Let $\mathcal{L}^{\diamond}$ be the corresponding modal language, and let $\vdash^{\diamond}$ be deducibility in this language corresponding by $\vdash, \mathrm{S} 4.2$, and axioms asserting the stability of all atomic predicates of $\mathcal{L}$.

Theorem 1 (Classical potentialist mirroring) For any formulas $\phi_{1}$, $\ldots, \phi_{n}, \psi$ of $\mathcal{L}$, we have:

$$
\phi_{1}, \ldots, \phi_{n} \vdash \psi \quad \text { iff } \quad \phi_{1}^{\diamond}, \ldots, \phi_{n}^{\diamond} \vdash^{\diamond} \psi^{\diamond} .
$$

(See [33] for a proof.)

The assumptions of the theorem were defended in Section 4, namely a modal logic based on $\mathrm{S} 4.2$ and the stability axioms. It is worth observing that the use of S4.2, rather than just S4, is essential. Without the assumption that the accessibility relation is directed and thus that the axiom $\mathrm{G}$ is valid, the theorem would no longer hold. ${ }^{20}$

The theorem has a simple moral. Suppose we are interested in logical relations between the modalized translations, in a classical modal theory that includes S4.2 and the stability axioms. Then we may delete all the modal operators and proceed by the ordinary non-modal logic underlying $\vdash$. In particular, under the stated assumptions, the modalized quantifiers $\square \forall$ and $\diamond \exists$ behave logically just as ordinary quantifiers, except that they generalize across all (accessible) possible worlds rather than a single world. This buttresses our choice of the potentialist translation as the bridge connecting actualist and potentialist theories.

The most important upshot of the theorem, however, is that ordinary classical first-order logic is validated via this bridge. This answers one of our guiding questions. Consider an Aristotelian notion of potential infinity. We take it this notion is based on some form of metaphysical modality, which behaves classically. Given this and the fact that Aristotle does not seem to allow any exceptions to the Law of Excluded

\footnotetext{
${ }^{20}$ For instance, while $\exists x \phi(x) \wedge \exists x \neg \phi(x)$ entails $\exists x \exists y(x \neq y)$, the potentialist translation of the former does not entail that of the latter. We see this by considering a Kripke model with three worlds, where $w_{0}$ has access to $w_{1}$ and $w_{2}$, but no other world has access to any world other than itself. Let the domain of $w_{1}$ and $w_{2}$ contain a single object, which satisfies $\phi$ at $w_{1}$ but not at $w_{2}$.
} 
Middle, he - and all the thinkers he inspired - are entitled to take the logic of potential infinity to be classical. ${ }^{21}$

It might be objected that on this liberal explication, potential infinity is scarcely different from actual infinity. Consider the case of arithmetic. While actualists defend classical first-order Dedekind-Peano arithmetic, liberal potentialists defend a theory that is equivalent via the mirroring theorem. One response is that, while the theories are indeed equivalent in this sense, their intended interpretations are very different: one involves modal notions, which the other eschews. ${ }^{22}$

The robustness of this response depends on the robustness of our grasp on the modality. When the modality is metaphysical, as it is for our Aristotelian, most contemporary philosophers would grant that there is a real difference. When the modality is understood in some other way, however, it needs to be confirmed that the modality makes a real difference and that the resulting theory isn't just actualism in potentialist garb. A second, supplementary response is developed in the next section, where we show that actualism and liberal potentialism validate different principles of higher-order logic.

Suppose, now, that potentialism pushes in the direction of intuitionistic logic. If so, this push must be compatible with the potentialist translation. How might this work? As a first step, consider what happens when the propositional modal logic S4.2 is combined with intuitionistic rather than classical first-order logic.

Our question is answered by another mirroring theorem. As usual, we say that a formula $\Phi$ is decidable in a given theory if the universal closure of $\Phi \vee \neg \Phi$ is deducible in that theory. Let $\vdash_{\text {int }}$ be the relation of intuitionistic deducibility in a first-order language $\mathcal{L}$, and let $\vdash_{\text {int }}^{\diamond}$ be deducibility in the modal language corresponding to $\mathcal{L}$, by $\vdash_{\text {int }}, \mathrm{S} 4.2$, the stability axioms for all atomic predicates of $\mathcal{L}$, and the decidability of all atomic formulas of $\mathcal{L}$.

\footnotetext{
${ }^{21}$ In a short appendix to an article on formalism, Abraham Robinson [45] defines a notion of "potential truth" for a language of arithmetic. He is keen to show that mathematics does not require the actual existence of anything infinite. Robinson does not use a modal language, but he does develop a Kripke-style framework (not under that name). Robinson shows that potential truth is the the same (in extension) as regular truth on the full domain (i.e., the union of the "worlds"). He has what is, in effect, a directed frame (where any two nodes have a common "future"). And his definitions include the positive and negative stability of the atomics. One can "translate" his clauses (for potential truth) into that of the corresponding modal language. If rendered into the modal framework, Robinsion's clause for $\exists x \Phi$ is $\diamond \exists x \square \Phi$.

${ }^{22}$ The potentialist can use the background modal language to state certain metaphysical principles that have no non-modal translation. See, for example, [35].
} 
Theorem 2 (Intuitionistic potentialist mirroring) For any formulas $\phi_{1}, \ldots, \phi_{n}, \psi$ of $\mathcal{L}$, we have:

$$
\phi_{1}, \ldots, \phi_{n} \vdash_{\text {int }} \psi \quad \text { iff } \quad \phi_{1}^{\diamond}, \ldots, \phi_{n}^{\diamond} \vdash_{\text {int }}^{\diamond} \psi^{\diamond} .
$$

(See Appendix B for a proof.)

Together, the two mirroring theorems show how our analysis of quantification over a potentially infinite domain can be separated from the question of whether the appropriate logic is classical or intuitionistic. Hold fixed our modal analysis of potential infinity, the propositional modal logic S4.2, and the potentialist bridge. Then the appropriate logic of potential infinity depends entirely on the first-order logic used in the modal system. Whichever first-order logic we plug in on the modal end - classical or intuitionistic - we also get out on the non-modal end. Since liberal potentialists see no reason to plug in anything other than classical first-order logic, they can reasonably regard this as the correct logic for potential infinity.

It is far less clear what strict potentialists should say, given their added requirement that every truth be "made true" at some stage. We later ask whether they have reason to take the logic of the modal language to be intuitionistic, which would entail that the logic of potential infinity too is intuitionistic.

\section{The higher-order logic of potential infinity}

The only difference between actualism and liberal potentialism that we have detected so far is a philosophical one, concerned with the presence or absence of an implicit modal aspect of ordinary mathematical quantification. Both approaches are entitled to classical first-order logic in the non-modal language of ordinary mathematics. However, an interesting technical difference emerges when we consider higher-order logic. This fact is significant, since many mathematical theories use higher-order axioms: induction for arithmetic, completeness for geometry, Dedekind or Cauchy completeness for real analysis, replacement for set theory. ${ }^{23}$ These principles are usually formulated using some form of higher-order variables.

Our procedure is as before. First, we identify the appropriate higherorder modal logic to be applied to our analysis of potential infinity. Then, we examine which non-modal inferences are validated via the potentialist bridge.

\footnotetext{
${ }^{23}$ With the exception of replacement, the various principles can be formulated in terms of sets. That would push our issues to the proper formulation of set theory, especially when some of the sets are infinite.
} 
We remind the reader that the above mirroring theorems are stated only for first-order languages. It is straightforward to extend them to the standard introduction and elimination rules for higher-order quantifiers, formulated in terms of free higher-order variables or parameters. The issues concern the comprehension scheme. In other words, we must figure out which formulas determine higher-order entities.

There are two kinds of extension of our first-order system to consider: to plural logic, which permits quantification into plural noun phrase position; and to second-order logic, which permits quantification into predicate position, in effect quantification over properties. In the usual extensional contexts of classical mathematics, these two approaches are substantially equivalent. No so here. We begin with the plural interpretation, where the most interesting and clear cut difference between actualists and even liberal potentialists emerges.

Let us first remind ourselves how plural logic without modality can be axiomatized. Of course, given the incompleteness of plural and secondorder logics with respect to standard semantics, the axiomatization cannot be complete. But there is a standard axiomatization, which suffices for a wide range of deductive purposes. We begin by adopting the ordinary introduction and elimination rules for the plural quantifiers. Next, we adopt the ordinary unrestricted plural comprehension scheme:

(P-Comp) $\exists x \phi(x) \rightarrow \exists x x \forall u[u \prec x x \leftrightarrow \phi(u)]$

That is, provided there is at least one $\phi$, there are some things $x x$ that are all and only the $\phi$ 's.

Now we wish to add modality to the mix. We do this in the same straightforward way as in the singular first-order case. This means that we can prove a plural version of the converse Barcan Formula. But this is unproblematic for the same reason as before, namely that all of our domains are non-decreasing along the accessibility relation. We can also allow each plural comprehension axiom to be necessitated. In any world, any instantiated condition $\phi(x)$ can be used to define some things that are - that is, are at the relevant world - all and only the $\phi$ 's.

What about the modal behavior of plural membership? In this context, we contend it is plausible to take pluralities to be modally rigid. That is, when $x$ is one of some objects $y y$, then this is necessarily so, at least on the assumption of the continued existence of $y y$. And likewise when $x$ is not one of $y y$. Since our intended interpretation has domains that are non-decreasing along the accessibility relation, we may omit the existential presuppositions and adopt the following rigidity principles for 
plural membership: ${ }^{24}$

$$
x \prec y y \rightarrow \square(x \prec y y) \quad x \nprec y y \rightarrow \square(x \nprec y y)
$$

Modal rigidity is quite intuitive, at least for metaphysical modality, and for some plural constructions, such as lists. Consider Barack and Michelle. If Michelle were not one of some people, then these people would not be Barack and Michelle but some other people. Likewise, if Vladimir were one of some people, these people would not be Barack and Michelle but some other people. Modal rigidity has, admittedly, been challenged as an interpretation of the ordinary, English plural idiom in full generality (see [20]). But for present purposes, we would, if necessary, be content simply to stipulate the ridigidy. The alternative treats plurals as more intensional, like properties. They would thus fall under our treatment of second-order logic.

We are now ready to determine the appropriate plural logic for reasoning about a potentially infinite domain. Suppose we reason plurally about such a domain but leave the modality implicit. Under what conditions do some formulas $\phi_{1}, \ldots, \phi_{n}$ entail $\psi$ ? To answer the question, we must first make the modality explicit - which we do by applying the potentialist translation. The answer is thus: when and only when $\phi_{1}^{\diamond}, \ldots, \phi_{n}^{\diamond}$ entail $\psi^{\diamond}$ in our modal system.

By far the most interesting case is the plural comprehension scheme. Which of its instances remain valid when applied to a potentially infinite domain? To answer the question, we apply the potentialist translation to obtain:

$$
\left(\mathrm{P}-\mathrm{Comp}^{\diamond}\right) \diamond \exists x \phi(x) \rightarrow \diamond \exists x x \square \forall u[u \prec x x \leftrightarrow \phi(u)]
$$

So when properly explicated, the question is this: for which formulas $\phi$ that are possibly instantiated is it possible for there to be some objects $x x$ which necessarily are all and only the $\phi$ 's?

The rigidity of pluralities gives rise to a surprising answer. Consider the condition ' $x=x$ ' of being self-identical. Since the condition is obviously instantiated, the question is whether it is possible for there to

\footnotetext{
${ }^{24}$ The latter principle ensures that a plurality can never grow at a larger world by picking up members that were antecedently available. We would also like to state that a plurality cannot grow by picking up members that became available only at the larger world. It turns out this can be expressed by the following axiom scheme:

$$
(\forall x \prec y y) \square \theta \rightarrow \square(\forall x \prec y y) \theta
$$

See Linnebo [33] for explanation. (Hint: the axioms are Barcan formulas for the relativized quantifier $\forall x \prec y y$.)
} 
be some objects $x x$ which necessarily are all and only the self-identical objects, that is, such that:

$$
\square \forall u(u \prec x x \leftrightarrow u=u)
$$

The answer is 'no'. By the rigidity of pluralities, the condition of being one of $x x$ is rigid and therefore not satisfied by more objects as we consider more populous possible worlds. By contrast, the condition of being self-identical is necessarily satisfied by everything and thus must be satisfied by more objects as we consider more populous worlds. It follows that the two conditions cannot be necessarily coextensive, and hence that the corresponding instance of $\left(\mathrm{P}-\mathrm{Comp}^{\diamond}\right)$ must be rejected.

So potentialists of all stripes have a reason to restrict the plural comprehension scheme. So here we have a clear logical difference between actualism, on the one hand, and the two forms of potentialism, on the other.

Consider, for example, the theory of the natural numbers and pluralities thereof. Since actualists are entitled to unrestricted plural comprehension, their version of the theory is in effect full classical second-order PA (the only difference being the trivial one that there is no empty class of numbers, as all pluralities must be non-empty). By contrast, a traditional Aristotelian potentialist is committed to the view that all pluralities are finite. This motivates a plural variant of so-called weak second-order logic, where the second-order variables are stipulated to range over all and only finite collections from the first-order domain (see [48], Chapter 9).

Unlike plural logic, which generalizes into plural noun-phrase position, second-order logic (as we will henceforth use the term) generalizes into predicate position. What do our various conceptions of the infinite entail concerning second-order logic? Our answer proceeds much as in the case of plural logic. So we limit ourselves to pointing out the differences. Unlike the semantic value of a plural noun-phrase, which we take to be modally rigid, there is no reason to expect the semantic value of all predicates to be rigid. ${ }^{25}$ For example, although Socrates satisfies the predicate 'is a philosopher', he might not have done so. Thus, the considerations that required potentialists to restrict plural comprehension are not available in the case of second-order logic. Consider the second-order comprehension scheme:

(2-Comp) $\exists F \forall x(F x \leftrightarrow \phi(x))$

\footnotetext{
${ }^{25}$ Of course, some predicates will have rigid semantic values; for example, the predicate ' $x=$ Socrates'.
} 
Applying the translation (to all but the embedded formula $\phi$ ), the two potentialists hold that the question can be explicated as follows ${ }^{26}$ :

$\left(2-\mathrm{Comp}^{\diamond}\right) \diamond \exists F \square \forall x(F x \leftrightarrow \phi(x))$

There is no obvious reason why the liberal potentialist should wish to restrict this. Since the concept $F$ need not be modally rigid, it is fine to let its application condition at any possible world be given by the condition $\phi(x)$ at that world, even if the embedded formula $\phi$ contains modal operators. Since this potentialist is liberal, she has every reason to assume the condition expressed by $\phi$ to yield a determinate truthcondition at every world. So on the question of second-order logic, there is no reason why the liberal potentialist should disagree with the actualist. Near the end of this paper, we briefly consider the question of what the strict potentialist should say about second-order logic.

In sum, liberal potentialists have a reason to restrict higher-order comprehension on the plural interpretation but not on the conceptual or second-order interpretation.

What is the significance of this discovery? In cases where the interpretation of the higher-order variables doesn't matter, this means there is no deep logical difference between actualism and liberal potentialism. Both are entitled to a form of full second-order arithmetic, for example, provided that the second-order variables are interpreted conceptually.

There may, however, be cases where it does matter that the higherorder variables are interpreted plurally. A case in point is the question of which "collections" define sets. ${ }^{27}$ An analogy conveys the gist of the argument. Suppose we are designing a web page. To which "collections" of web pages may our new page be required to link? The answer depends on how the "collection" is specified. If it is specified intensionally by the concept web page that doesn't link to itself, it would be paradoxical to require our new page to link to all and only members of that "collection". The new page would have to link to itself just in case it does not link to itself. By contrast, if the "collection" is specified extensionally by the plurality of each and every web page that doesn't link to itself, it is unproblematic to require our new web page to link to all and only members of that "collection". According to the argument in question, the question of set formation is analogous to the question of web page

\footnotetext{
${ }^{26}$ Some may want to drop the opening possibility operator. This depends on the modal status of properties and relations. Do they exist at some worlds and not others (so to speak), or are they somehow outside of the realm of possible worlds - existing of necessity? We will say no more on this matter of metaphysics.

${ }^{27}$ See Linnebo [32].
} 
design: while there is no compelling reason to think that every concept defines a set, it is hard to resist the view that every plurality suffices to define a set. This view of set formation traces its roots back to Cantor, whose distinction between "consistent" and "inconsistent multiplicities" (cf. Section 2) mirrors ours between pluralities and mere concepts. Our discussion in the present section shows that this view of set formation is available to liberal potentialists but not to actualists.

\section{Strict potentialism}

Strict potentialism, we recall, goes beyond its liberal cousin by requiring, not only that every object be generated at some stage of a process, but also that every truth be "made true" at some stage. For example, "18 is the sum of two primes" is plausibly taken to be "made true" once the numbers up through 18 have been generated. By that stage, we have established that $5+13=18$ and $7+11=18$. There is no need to look beyond these five numbers to determine the truth of our example. For an existential generalization is plausibly "made true" when a witness is generated. ${ }^{28}$ Next, consider the claim that there are counterexamples to Goldbach's conjecture, that is, even numbers that are not the sum of two primes. If true, this claim is presumably "made true" when the first counterexample is generated.

By contrast, universal generalizations over the natural numbers pose a problem for strict potentialists. Again, consider Goldbach's conjecture. Since the conjecture is concerned with all the natural numbers, it is hard to see how it could be "made true" at any finite stage where, after all, only finitely many numbers will have been generated. Yet if we are serious about the merely potential character of the sequence of natural numbers, strict potentialists contend, there cannot be any arithmetical truths that are "made true" only by the sequence in its entirety. When a sequence is incompletable, there is no such thing as "the sequence in its entirety". In short, the extra demand that differentiates strict from liberal potentialism puts great pressure on universal generalizations over a potentially infinite domain. It is hard to see how a generalization over all F's could be "made true" by some particular stage at which most $F$ 's haven't even been generated.

For strict potentialism to provide a coherent conception of potential infinity, two challenges need to be met. First, the loose talk about being "made true" needs to be made formally precise. Second, we need to explain how strict potentialists can make sense of universal generalizations over potentially infinite collections, such as the natural numbers.

\footnotetext{
${ }^{28}$ We say that $a$ is a witness to $\exists x \phi(x)$ at a world $w$ iff $\phi(a)$ is "made true" at $w$.
} 
Without the ability to state and prove such generalizations, we do not have a conception of the infinite, only a draconian restriction of quantificational reasoning to finite domains. Our aim in what follows is to show how both challenges can be met and how this implies that the logic of potential infinity is intuitionistic. For example, we explain what it is for Goldbach's conjecture to be "made true" at some finite stage, despite the conjecture's concern with numbers not generated by that stage. But on this account there is no guarantee that either the conjecture or its negation will be made true by any finite stage - thus the connection to excluded middle and intuitionistic logic.

What might it be for a universal generalization over the natural numbers to be "made true" at some finite stage? Traditional intuitionism proposes one answer, namely to identify mathematical truth with our possession of a proof, or at least of an algorithmic strategy guaranteed to yield one. ${ }^{29}$ On this strong anti-realist conception of truth, a generalization is "made true" when we produce a proof of it. And since every proof is produced at some finite stage, this satisfies the strict potentialist's requirement. ${ }^{30}$ The problem is the strong anti-realism on which this answer rests. The generative process is understood as a process of actual constructions, whereby mathematical objects and truths/proofs - which did not previously exist or obtain - are brought into being.

Thankfully, another answer is available, which avoids saddling strict potentialism with the controversial anti-realist views of traditional intuitionism. We take our cue from Hermann Weyl ([54], 54), who writes in a discussion of whether there is a natural number that has some decidable property $P$ as follows.

Only the finding that has actually occurred of a determinate number with the property $P$ can give a justification for the answer "Yes," and - since I cannot run a test through all numbers - only the insight, that it lies in the essence of number to have the property not- $P$, can give a justification for the answer "No"; Even for God no other ground for decision is available.

On this view, the truth of the universal generalization - that every number is not- $P$ - has nothing to do with epistemic matters, such as our knowledge or proofs. Even God, who is assumed to know all the facts, cannot know facts that require running through all the natural numbers.

\footnotetext{
${ }^{29}$ This qualification will henceforth be elided.

${ }^{30}$ Brouwer's treatment of the creative subject goes along these lines.
} 
The point is rather that there are no such facts! When a universal generalization is true, it is instead made true by its lying "in the essence number" to have the relevant property.

Weyl's proposal, as we understand it, is that not every generalization is "made true" by the totality of its instances. Consider the truths that every red object is colored and that every atom of gold consists of 79 protons. These truths seem unconcerned with individual red objects or atoms of gold. They seem to be "made true" not by their instances but by what it is to be red or colored. Likewise, we propose, there are essence-based constraints on any future generation of the objects studied by mathematics. For example, it is a constraint on the generation of natural numbers that the arithmetical successor operation be injective:

$$
\operatorname{SuCC}\left(x, x^{\prime}\right) \wedge \operatorname{SuCC}\left(y, y^{\prime}\right) \wedge x^{\prime}=y^{\prime} \rightarrow x=y
$$

This important arithmetical axiom is "made true" by the mentioned constraint prior to the generation of any particular natural numbers.

Admittedly, these are deep metaphysical waters, even if not exactly those of orthodox intuitionism. In what follows, we explain the ideas in question and provide at least one precise mathematical model. Our aim is to meet the two challenges discussed above in a way that does not rely on any anti-realist ideas, such as an identification of mathematical truth with our possession of a proof. A striking feature of our explication of strict potentialism is that it leads to intuitionistic logic. This means that we provide a route from strict potentialism to intuitionistic logic that is independent of any form of anti-realism.

\section{A mathematical model of truthmaking}

In the case of arithmetic, at least, a good first approximation is provided by the realizability interpretation, going back to Stephen Cole Kleene [24], in 1945. The loose talk about what "lies in the essence of number" can be understood in terms of computable functions.

Let $\{e\}(n)$ be the result of applying Turing machine with index $e$ to the input $\bar{n}$. We now define what it is for a natural number $e$ to be a realizer for a sentence $\phi$, written $e \Vdash \phi$. The idea is that $e$ encodes information that establishes the truth of $\phi$. For present purposes, a useful metaphysical heuristic is that $e$ functions as a "truth maker" for $\phi$.

The most important clause is the one for the universal quantifier, where we define

$$
e \Vdash \forall n \phi(n) \quad \text { iff } \quad \forall n\{e\}(n) \Vdash \phi(n)
$$


That is, $e$ realizes the universal generalization $\forall n \phi(n)$ just in case the Turing machine $\{e\}$ computes a realizer for the instance $\phi(n)$ when given any numeral $\bar{n}$ as input. In terms of our metaphysical heuristic: $e$ is a truth maker for $\forall n \phi(n)$ just in case $e$ specifies a function that maps any numeral $\bar{n}$ to a truth maker for the associated instance $\phi(n)$.

Let's now regard a formula as true just in case it has a realizer or "truth maker". Since a realizer is just a natural number, this means that any true formula is made true after finitely many steps. So our strict potentialist avoids having to wait until the end of time.

Of course, there remains the question of whether this definition yields the right truths. For our strict potentialist, a natural measure of what is "right" is provided by the standard intuitionist theory of arithmetic, known as Heyting arithmetic, whose axioms as the same as those of first-order Peano-Dedekind arithmetic but where the logic is intuitionistic, not classical. Pleasingly, there is a theorem stating that Heyting arithmetic is sound with respect to the notion of truth that we have defined.

Theorem 3 (Realizability) Every theorem of Heyting arithmetic has a realizer. However, there are theorems of first-order Dedekind Peano arithmetic that do not have a realizer.

Not every sentence with a realizer is a theorem of Heyting arithmetic, however. For example, the intuitionistic version of Church's thesis has a realizer, although Church's thesis is not a theorem of Heyting arithmetic. $^{31}$

Let us put all the pieces together. All we are entitled to assume concerning truth making at a world is intuitionistic logic. Let us now kick away the ladder of this talk about worlds in favor of just the modal operators, representing the generative modality and based on $\mathrm{S} 4.2$. It follows that this logic must be based on intuitionistic logic, not classical. Thus, for the strict potentialist, the appropriate modal system is one based on intuitionistic S4.2.

\footnotetext{
${ }^{31}$ This version of Church's thesis is inconsistent with classical arithmetic. Troelstra [52], $\S 1.11$, shows how to formulate realizability within the language of arithmetic, and gives a sharp result. $\mathrm{HA}^{*}$ is a conservative extension of Heyting arithmetic, augmented with a term for the application of partial recursive functions, and $\mathrm{ECT}_{0}$ is a slight extension of Church's thesis. Then $\mathrm{HA}^{*}$ plus $\mathrm{ECT}_{0}$ entails that any formula $A$ is equivalent to the statement that $A$ has a realizer. Also, for any sentence $A$, classical Dedekind-Peano arithmetic proves that $A$ has a realizer if and only if Heyting arithmetic plus $\mathrm{ECT}_{0}$, plus Markov's principle proves $\neg \neg A$. Markov's principle is that if a given formula $B$ is decidable, then $\neg \neg \exists x B \rightarrow \exists x B$.
} 
As before, the next step is to apply the potentialist translation to provide a bridge back to the non-modal language of ordinary mathematics. The result of doing so has already been established by Theorem 2, on intuitionistic potentialist mirroring. As we recall, the theorem ensures the following:

$$
\phi_{1}, \ldots, \phi_{n} \vdash_{\text {int }} \psi \quad \text { iff } \quad \phi_{1}^{\diamond}, \ldots, \phi_{n}^{\diamond} \vdash_{\text {int }}^{\diamond} \psi^{\diamond} .
$$

The bottom line is that, for the strict potentialist, the appropriate firstorder logic for reasoning about a potentially infinite domain is intuitionistic. This confirms a claim by Dummett and others, which has so far never been properly substantiated, namely that only intuitionistic quantification, not classical, is permitted over a domain that is potentially infinite (or "indefinitely extensible") - provided the potentiality is strict.

We end this section with some very brief remarks about higher-order logic from a strict potentialist point of view. Note, first, that the results concerning realizability are restricted to first-order languages. So, for now, we must restrict comprehension to those instances in which the embedded formula is first-order. Any attempt to go beyond this would require extending realizability to higher-order languages, which goes beyond the scope of this article.

Second, recall that the intuitionistic mirroring theorem requires all atomic predicates to be decidable. This means that the "membership" notions $x \prec y y$ and $F x$ of plural and second-order logic, respectively, need to be decidable. This will obviously result in a further restriction on comprehension axioms. Consider second-order comprehension, which the liberal potentialist can accept in its classical, unrestricted form. This must now be restricted as follows:

$$
\forall x(\phi(x) \vee \neg \phi(x)) \rightarrow \exists F \forall x(F x \leftrightarrow \phi(x))
$$

An analogous restriction must be added to plural comprehension scheme (over and above the restrictions already imposed by liberal potentialists).

Summing up, we have described a theoretically interesting conception of arithmetical truth, which satisfies the strict potentialist's requirement that every truth be made true after some finite number of steps, and on which all the theorems of first-order intuitionistic-but not classicalarithmetic are true. Notice, again, that the real locomotive of the argument is strict potentialism. There is no direct reliance on an anti-realist conception of the numbers.

\section{Conclusions}

Let us return to William Tait's questions, mentioned in the introduction. He first observed that Hilbert and the early intuitionists associated a 
commitment to the actual infinite with the use of classical logic. For example, they thought that the use of quantification over the natural numbers combined with classical logic commits one to the set of natural numbers as an actual infinity. Tait then writes:

I would like someone to explain why this is the same notion of actual infinity as Aristotle's. (One might ask, too, whether quantification over the integers using intuitionistic logic commits one to the actual infinite - and why.)

Of course, we are not scholars of the early history of mathematics and philosophy. But we claim that the accounts of potential infinity developed here are in the spirit of the Aristotelian notion of potential infinity, using contemporary modal logic (in roughly the same spirit as that of [55]). In the course of this article, we have answered the questions prompted by Tait.

(A) If the natural numbers are merely potentially infinite, are we entitled to quantify over all of them using (at least) intuitionistic logic?

The answer is affirmative. First, we showed that liberal potentialismwhich is arguably the version of potentialism closest to Aristotle'ssanctions such quantification subject to classical logic. Then, we examined the strict potentialist's added requirement that every truth be "made true" at some finite stage of the generative process. We showed that this is modeled by the realizability interpretation, which sanctions such quantification subject to intuitionistic logic.

Next:

(B) Does quantification over all the natural numbers with classical logic presuppose actual infinity?

Here the liberal and the strict potentialist part company. The liberal answers the question negatively, insisting we are entitled to classical quantification without assuming any actual infinities. The classical logic is sanctioned by the liberal's realist attitude toward the underlying modality. The strict potentialist disagrees, insisting that the potential infinity of the natural numbers removes the licence to anything stronger than intuitionistic quantification.

In the course of our discussion, we also found that our three main characters - the actualist, and the liberal and strict potentialists - have interestingly different commitments concerning higher-order logics as well. The following diagram summarizes our findings. 


\begin{tabular}{|c|c|c|c|c|}
\hline & face value & classical FOL & 2-Comp & P-Comp \\
\hline ACTUALISM & $\checkmark$ & $\checkmark$ & $\checkmark$ & $\checkmark$ \\
\hline LIBERAL POT. & $x$ & $\checkmark$ & $\checkmark$ & $x$ \\
\hline Strict Pot. & $x$ & $x$ & $x$ & $x$ \\
\hline
\end{tabular}

The columns represent whether the non-modal language of ordinary mathematics can be taken at face value; whether to accept classical firstorder logic (as opposed to intuitionistic); whether to accept unrestricted second-order comprehension; and whether to accept unrestricted plural comprehension. 


\section{Appendices}

\section{A The Gödel translation}

The Gödel translation $\dagger$ is given by the following clauses:

$$
\begin{aligned}
\phi & \mapsto \square \phi \quad \text { for } \phi \text { atomic } \\
\neg \phi & \mapsto \square \neg \phi^{\dagger} \\
\phi \vee \psi & \mapsto \phi^{\dagger} \vee \psi^{\dagger} \\
\phi \wedge \psi & \mapsto \phi^{\dagger} \wedge \psi^{\dagger} \\
\phi \rightarrow \psi & \mapsto \square\left(\phi^{\dagger} \rightarrow \psi^{\dagger}\right) \\
\forall x \phi & \mapsto \square \forall x \phi^{\dagger} \\
\exists x \phi & \mapsto \exists x \phi^{\dagger}
\end{aligned}
$$

Let $\vdash_{\mathrm{S} 4}$ be deducibility in the logic that results from combining S4 with classical first-order logic. Then we have the following theorem.

Theorem 4 Let $\vdash_{\text {int }}$ be intuitionistic deducibility in the given language. Let $\vdash_{\mathrm{S} 4}$ be the corresponding deducibility relation in S4 and classical logic. Then we have:

$$
\phi_{1}, \ldots, \phi_{n} \vdash_{\text {int }} \psi \quad \text { iff } \quad \phi_{1}^{\dagger}, \ldots, \phi_{n}^{\dagger} \vdash_{\mathrm{S} 4} \psi^{\dagger} .
$$

\section{B Mirroring for intuitionists}

We use the framework due to Alex K. Simpson [50]. We work in a more or less standard possible-worlds-framework for the modal operators. We define the notion of frame and interpretation, giving the usual clauses for the connectives and quantifiers (i.e., not the ones in the usual Kripke framework for intuitionism). ${ }^{32}$ We use intuitionistic logic in the metatheory (for the most part).

We begin by establishing an analogue of Lemma 5.3 of [33]:

Lemma 1 Let $\Phi$ be a fully modalized formula in a modal language. Then intuitionistic S4.2, the stability axioms for that language, and the decidability of all atomic formulas, prove that $\diamond \Phi, \Phi$, and $\square \Phi$ are equivalent.

\footnotetext{
${ }^{32}$ So we say, for example, that a formula $\forall x \Phi(x)$ is true at a world $w$ just in case $\Phi(a)$ is true in $w$ for all objects $a$ in the domain of $w$. This, of course, is different from the clause for the universal quantifier in the Kripke semantics for intuitionistic logic (which requires $\Phi$ to be true for all objects in all worlds accessible from $w$ ).
} 
Proof. Clearly, it suffices to show that if $\Phi$ is fully modalized, then $\diamond \Phi \rightarrow \square \Phi$ holds. The proof goes by induction on the complexity of $\Phi$. Suppose, first, that $\Phi$ is atomic. Then, by hypothesis, $\Phi$ is decidable: Suppose $\diamond \Phi$. We also have $\Phi \vee \neg \Phi$. Argue by cases. If $\Phi$ then, by stability, we have $\square \Phi$, and we are done. If $\neg \Phi$, then, by stability $\square \neg \Phi$. But this is inconsistent with the hypothesis $\diamond \Phi$.

So suppose the Lemma holds for $\Phi$ and for $\Psi$. Suppose that $\diamond \neg \Phi$ holds at a given world $w$. We want to show that $\square \neg \Phi$ also holds at $w$. So suppose $w \leq w^{\prime}$. Suppose, for reductio, that $\Phi$ holds at $w^{\prime}$. Then, by the induction hypothesis, $\square \Phi$ holds at $w^{\prime}$. Since $\diamond \neg \Phi$ holds at $w$, there is a world $w^{\prime \prime}$ such that $w<w^{\prime \prime}$ and $\neg \Phi$ holds at $w^{\prime \prime}$. By the contrapositive of the induction hypothesis, $\neg \diamond \Phi$ holds at $w^{\prime \prime}$. By directness, there is a world $w^{\prime \prime \prime}$ such that $w^{\prime} \leq w^{\prime \prime \prime}$ and $w^{\prime \prime} \leq w^{\prime \prime \prime}$. By the former, we have that $\Phi$ holds at $w^{\prime \prime \prime}$ and so $\diamond \Phi$ holds at $w^{\prime \prime}$. This is a contradiction. So $\Phi$ does not hold at $w^{\prime}$. So $\neg \Phi$ holds at $w^{\prime}$. Since $w^{\prime}$ is arbitrary, $\square \neg \Phi$ holds at $w$.

Suppose next that $\diamond(\Phi \& \Psi)$. Then $\diamond \Phi \& \diamond \Psi$. So, by the induction hypothesis, $\square \Phi \& \square \Psi$. So $\square(\Phi \& \Psi)$.

Suppose now that $\diamond(\Phi \vee \Psi)$. Then $\diamond \Phi \vee \diamond \Psi$. So, by the induction hypothesis, $\square \Phi \vee \square \Psi$. So $\square(\Phi \vee \Psi)$.

Suppose now that $w$ is a world in an interpretation such that $\diamond(\Phi \rightarrow$ $\Psi)$ holds at $w$. We want to show that $\square(\Phi \rightarrow \Psi)$ holds at $w$. So suppose that $w \leq w^{\prime}$ and $\Phi$ holds at $w^{\prime}$. We want to show that $\Psi$ holds at $w^{\prime}$. We have that $\diamond \Phi$ holds at $w$. So, by the induction hypothesis, $\square \Phi$ holds at $w$. We have that $\diamond(\Phi \rightarrow \Psi)$ holds at $w$. So there is a world $v$ such that $w \leq v$ and $(\Phi \rightarrow \Psi)$ holds at $v$. Also $\Phi$ holds at $v$ (since $\square \Phi$ holds at $w)$. So $\Psi$ holds at $v$. And so $\diamond \Psi$ holds at the original world $w$. By the induction hypothesis, $\square \Psi$ holds at $w$. So $\Psi$ holds at $w^{\prime}$ (since $w \leq w^{\prime}$ ). So $(\Phi \rightarrow \Psi)$ holds at $w^{\prime}$ (discharging the assumption that $\Phi$ holds at $w$ ). But $w^{\prime}$ is arbitrary. So $\square(\Phi \rightarrow \Psi)$ holds at $w$.

Now suppose that the Lemma holds for each instance of $\Phi(x)$. We have to show that it holds for $\square \forall x \Phi(x)$. So assume that $\diamond \square \forall x \Phi(x)$ holds at a world $w$. We have to show that $\square \square \forall x \Phi(x)$ holds at $w$. This is the same as showing that $\square \forall x \Phi(x)$ holds at $w$. So suppose that $w \leq w^{\prime}$ and let $a$ be an object that exists at $w^{\prime}$ (so that $a \in D\left(w^{\prime}\right)$ ). By the (G) principle, we have that $\square \diamond \forall x \Phi(x)$ holds at $w$ and so $\diamond \forall x \Phi(x)$ holds at $w^{\prime}$. So there is a world $w^{\prime \prime}$ such that $w^{\prime} \leq w^{\prime \prime}$ and $\forall x \Phi(x)$ holds at $w^{\prime \prime}$. Since the domains grow (or do not shrink) along the accessibility relation, our object $a$ exists at $w^{\prime \prime}$, and so $\Phi(a)$ holds at $w^{\prime \prime}$. So $\diamond \Phi(a)$ holds at $w^{\prime}$. By the induction hypothesis, $\Phi(a)$ holds at $w^{\prime}$. Since $a$ was arbitrary, we have that $\forall x \Phi(x)$ holds at $w^{\prime}$. Since $w^{\prime}$ was arbitrary, we have that $\square \forall x \Phi(x)$ holds at $w$. And by $\mathrm{S} 4, \square \square \forall x \Phi(x)$ holds at $w$. 
Suppose, again, that the Lemma holds for each instance of $\Phi(x)$. We have to show that it holds for $\diamond \exists x \Phi(x)$. So assume that $\diamond \diamond \exists x \Phi(x)$ holds at a world $w$. In $\mathrm{S} 4$, this amounts to assuming that $\diamond \exists x \Phi(x)$ holds at $w$. We have to show that $\square \diamond \exists x \Phi(x)$ holds at $w$. So suppose that $w \leq w^{\prime}$. We have to show that $\diamond \exists x \Phi(x)$ holds at $w^{\prime}$. Since $\diamond \exists x \Phi(x)$ holds at $w$, there is a world $v$ such that $w \leq v$ and $\exists x \Phi(x)$ holds at $v$. So there is an object $a$ in $D(v)$ such that $\Phi(a)$ holds at $v$. By the induction hypothesis, $\square \Phi(a)$ holds at $v$. By convergence, there is a world $w^{\prime \prime}$ such that $w^{\prime} \leq w^{\prime \prime}$ and $v \leq w^{\prime \prime}$. Since $\square \Phi(a)$ holds at $v, \Phi(a)$ holds at $w^{\prime \prime}$. So $\exists x \Phi(x)$ holds at $w^{\prime \prime}$. So $\diamond \exists x \Phi(x)$ holds at $w^{\prime}$. Since $w^{\prime}$ is arbitrary, we have that $\square \diamond \exists x \Phi(x)$ holds at $w$.

Suppose that the Lemma holds for $\Phi$. We have to show that it holds for $\square \Phi$. So assume that $\diamond \square \Phi$ holds at a world $w$. By the $(\mathrm{G})$ principle, $\square \diamond \Phi$ holds at $w$. Let $w \leq w^{\prime}$. Then $\diamond \Phi$ holds at $w^{\prime}$. By the induction hypothesis, $\square \Phi$ holds at $w^{\prime}$. Since $w^{\prime}$ is arbitrary, $\square \square \Phi$ holds at $w$.

Suppose again (and finally) that the Lemma holds for $\Phi$. We have to show that it holds for $\diamond \Phi$. So assume that $\diamond \diamond \Phi$ holds at a world $w$ (to show that $\square \diamond \Phi$ holds at $w$ ). We thus have that $\diamond \Phi$ holds at $w$. By the induction hypothesis, $\square \Phi$ holds at $w$. Let $w \leq w^{\prime}$. So $\Phi$ holds at $w^{\prime}$. Since the accessibility relation is reflexive, we have that $\diamond \Phi$ holds at $w^{\prime}$. Since $w^{\prime}$ is arbitrary, we have that $\square \diamond \Phi$ holds at $w$. $\dashv$

We are ready to prove Theorem 2, namely the intuitionistic mirroring theorem. Start with the left-to right direction. As in [33], the proof goes by induction on the derivation. The only hard cases are the quantifier rules.

For the universal elimination rule, suppose we have $\Phi_{1}, \ldots, \Phi_{n}, \vdash$ $\forall x \Psi(x)$. The induction hypothesis gives us $\Phi_{1}^{\diamond}, \ldots, \Phi_{n}^{\diamond} \vdash^{\diamond} \square \forall x \Psi^{\diamond}(x)$, and from this conclusion we get $\forall x \Psi^{\diamond}(x)$ and thus $\Psi^{\diamond}(t)$.

For the universal introduction rule, suppose we have $\Phi_{1}, \ldots, \Phi_{n}, \vdash$ $\Psi(t)$, where the $t$ does not occur free in any of the premises. By the induction hypothesis, we have $\Phi_{1}^{\diamond}, \ldots, \Phi_{n}^{\diamond} \vdash^{\diamond} \Psi^{\diamond}(t)$. And so $\Phi_{1}^{\diamond}, \ldots, \Phi_{n}^{\diamond} \vdash^{\diamond}$ $\forall x \Psi^{\diamond}(x)$. A standard move in S4 gives us $\square \Phi_{1}^{\diamond}, \ldots, \square \Phi_{n}^{\diamond} \vdash^{\diamond} \square \forall x \Psi^{\diamond}(x)$. Our Lemma thus gives us $\Phi_{1}^{\diamond}, \ldots, \Phi_{n}^{\diamond} \vdash^{\diamond} \square \forall x \Psi^{\diamond}(x)$. The conclusion of this is $(\forall x \Psi(x))^{\diamond}$.

For the existential introduction rule, suppose we have $\Phi_{1}, \ldots, \Phi_{n}, \vdash$ $\Psi(t)$. The induction hypothesis gives us $\Phi_{1}^{\diamond}, \ldots, \Phi_{n}^{\diamond} \vdash^{\diamond} \Psi^{\diamond}(t)$, and so we have $\Phi_{1}^{\diamond}, \ldots, \Phi_{n}^{\diamond} \vdash \diamond \exists x \Psi^{\diamond}(x)$, and so $\Phi_{1}^{\diamond}, \ldots, \Phi_{n}^{\diamond} \vdash \diamond \diamond \exists x \Psi^{\diamond}(x)$.

Now for the existential elimination rule. Suppose we have

$$
\Phi_{1}, \ldots, \Phi_{n}, \Phi(t) \vdash \Psi
$$

where $t$ does not occur free in any of the $\Phi_{i}$, nor in $\Psi$. The induction hypothesis thus gives us $\Phi_{1}^{\diamond}, \ldots, \Phi_{n}^{\diamond}, \Phi^{\diamond}(t) \vdash^{\diamond} \Psi^{\diamond}$. That is, any world in 
which the premises hold, the conclusion holds as well (in that world). We have to show that $\Phi_{1}^{\diamond}, \ldots, \Phi_{n}^{\diamond}, \diamond \exists x \Phi^{\diamond}(x) \vdash^{\diamond} \Psi^{\diamond}$. So let $w$ be any world in which $\Phi_{1}^{\diamond}, \ldots, \Phi_{n}^{\diamond}$, and $\diamond \exists x \Phi^{\diamond}(x)$ all hold. We have to show that $\Psi^{\diamond}$ holds at $w$. Since $\diamond \exists x \Phi^{\diamond}(x)$ holds at $w$, there is a world $w^{\prime}$ such that $w \leq w^{\prime}$ and $\exists x \Phi^{\diamond}(x)$ holds at $w^{\prime}$. So there is an object $a$ in the domain of $w^{\prime}$ such that $\Phi^{\diamond}(a)$ holds at $w^{\prime}$. By the above Lemma, we have that $\square \Phi_{1}^{\diamond}, \ldots$, and $\square \Phi_{n}^{\diamond}$ all hold at $w$. So $\Phi_{1}^{\diamond}, \ldots$, and $\Phi_{n}^{\diamond}$ all hold at $w^{\prime}$. Now

recall that the induction hypothesis gives us $\Phi_{1}^{\diamond}, \ldots, \Phi_{n}^{\diamond}, \Phi^{\diamond}(t) \vdash^{\diamond} \Psi^{\diamond}$. The premises all hold at $w^{\prime}$, interpreting $t$ as $a$. So we have that $\Psi^{\diamond}$ holds at $w^{\prime}$. So, since $w \leq w^{\prime}$, we have that $\diamond \Psi^{\diamond}$ holds at the original world $w$. By the Lemma, we have that $\Psi^{\diamond}$ holds at $w$. This is what we were to show.

Now for the right-to-left direction. Take any derivation of

$$
\Phi_{1}^{\diamond}, \ldots, \Phi_{n}^{\diamond} \vdash^{\diamond} \Psi^{\diamond}
$$

and erase all of the boxes and diamonds, and all of the parameters for worlds. The result is easily converted in to an intuitionistic derivation of $\Phi_{1}, \ldots, \Phi_{n}, \vdash \Psi$.

\section{References}

[1] Annas, J., Aristotle's Metaphysics: Books $M$ and N, (Oxford, Clarendon Press, 1976).

[2] Aristotle, The Basic Works of Aristotle, R. McKeon, ed. (Random House, 1941)

[3] Arntzenius, Frank "Gunk, topology, and measure", in Oxford studies in metaphysics 4, edited by Dean W. Zimmerman (Oxford, Oxford University Press, 2008), 225-247.

[4] Barker, Chris, "Free choice permission as resource-sensitive reasoning", Semantics and Pragmatics 3 (2010), 1-38.

[5] Bohn, Einar Duenger, "Must there be a top level", Philosophical Quarterly 59 (2009), 193-201.

[6] Cantor, G., Grundlagen einer allgemeinen Mannigfaltigkeitslehre. Ein mathematisch-philosophischer Versuch in der Lehre des Unendlichen (Leipzig, Teubner, 1883).

[7] Cantor, G., "Mitteilugen zur Lehre vom Transfinitten 1, II"., Zeitschrift für Philosophie und philosophische Kritik 91 (1887), 81125, 252-270; 92, 250-265; reprinted in [8], 378-439.

[8] Cantor, G., Gesammelte Abhandlungen mathematischen und philosophischen Inhalts, edited by E. Zermelo (Berlin, Springer, 1932).

[9] Chomsky, Noam, Syntactic structures, The Hague, Mouton. 
[10] van Dalen, Dirk, "Intuitionistic logic", in The Blackwell Guide to Philosophica Logic, edited by L. Gobble, Oxford, Blackwell, 200, pp. 224257.

[11] Dummett, Michael, "The philosophical basis of intuitionistic logic", in Truth and other enigmas, by Michael Dummett, Cambridge Massachusetts, Harvard University Press, 1978, pp. 215-247

[12] Dummett, Michael, Frege: Philosophy of Mathematics (Harvard University Press, 1991).

[13] Euclid, The thirteen book of Euclid's Elements, translation and commentary by Thomas L. Heath, second revised edition (New York, Dover Publications, 1956).

[14] Ewald, William, editor, From Kant to Hilbert: a sourcebook in the foundations of mathematics (Oxford, Oxford University Press, 1996).

[15] Fine, Kit, "Our knowledge of mathematical objects", in Oxford studies in epistemology, Volume 1, edited by T. S. Gendler and J. Hawthorne (Oxford, Oxford University Press, 2005), pp. 89-110.

[16] Gauss, Karl Friedrich, "Briefwechsel mit Schumacher", Werke, Band 8, 216 (1831).

[17] Hellman, Geoffrey, Mathematics without Numbers: Towards a Modal-Structural Interpretation (Oxford University Press, 1989).

[18] Hellman, Geoffrey and Stewart Shapiro, "Towards a Point-free account of the Continuous", Iyyun: The Jerusalem Philosophical Quarterly 61 (2012): 263-287.

[19] Hellman, Geoffrey and Stewart Shapiro, "The classical continuum without points", Review of Symbolic Logic 6 (2013): 488-512.

[20] Hewitt, Simon, "Modalising plurals", Journal of Philosophical Logic 41 (2012): 853-875.

[21] Hilbert, David, Grundlagen der Geometrie (Leipzig, Teubner, 1899); translated by E. Townsend as Foundations of geometry, (La Salle, Illinois, Open Court, 1959).

[22] Hilbert, David, "Über das Unendliche", Mathematische Annalen 95 (1925), 161-190; translated as "On the infinite", in Philosophy of mathematics, second edition, edited by Paul Benacerraf and Hilary Putnam, Cambridge, Cambridge University Press, 1983, 183-201.

[23] Jané, Ignacio, 'Idealist and realist elements in Cantor's approach to set theory", Philosophia Mathematica (3) 18 (2010): 193-226.

[24] Kleene, Stephen Cole, "On the interpretation of intuitionistic number theory", Journal of Symbolic Logic 10 (1945): 109-124.

[25] Kripke, Saul, "Identity and necessity", in Identity and Individuation, edited by Milton K. Munitz (New York, New York University Press), pp. 135-164. 
[26] Lear, Jonathan "Aristotelian infinity", Proceedings of the Aristotelian Society, new series 80 (1980), 187-210.

[27] Lear, Jonathan, "Aristotle's philosophy of mathematics", Philosophical Review 41 (1982), 161-192.

[28] Leibniz, Gottfried, Mathematische Scriften von Gottfried Wilhelm Leibniz, edited by C. I. Gerhart (Berlin, A. Asher, HalleH. W. Schmidt, 1863).

[29] Leibniz, Gottfried, Samtliche Schriften und Briefe: Philosophishce Schriften, Series 6, Volumes 1-3, (Berlin, Akademie-Verlag, 19231980).

[30] Leibniz, Gottfried, New essays on human understanding, edited and translated by P. Remnant and J. Bennett (New York, Cambridge University Press, 1996).

[31] Levey, Samuel, "Leibniz on mathematics and the actually infinite

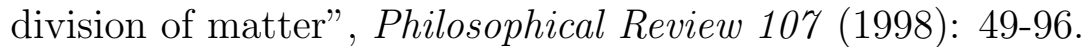

[32] Linnebo, Øystein, "Pluralities and sets", Journal of Philosophy 107 (2010): 144-164.

[33] Linnebo, Øystein, "The potential hierarchy of sets", Review of Symbolic Logic 6 (2013): 205-228.

[34] Linnebo, Øystein and David Nicolas, "Superplurals in English", Analysis 68 (2008): 186-197.

[35] Linnebo, Øystein, Stewart Shapiro, and Geoffrey Hellman, "Aristotelian continuua", Philosophia Mathematica (3): forthcoming.

[36] McCarty, Charles, "Paradox and potential infinity", Journal of Philosophical logic 42 (2013): 195-219.

[37] Miller, Fred D, "Aristotle against the atomists", in Infinity and continuity in ancient and medieval thought, edited by Normal Kretzmann (Ithaca, Cornell University Press, 2014), pp. 87-111.

[38] Niebergall, Karl-George "Is ZF finitistically reducible?", in One hundred years of Russell's paradox: mathematics, logic, philosophy, edited by Godehard Link, Berlin, Walter de Gruyter, 2004, pp. 153-180.

[39] Niebergall, Karl-George, "Assumptions of infinity", in Formalism and beyond: on the nature of mathematical discourse, edited by Godehard Link (Boston, De Gruyter), pp. 229-274.

[40] Parsons, Charles, "What is the iterative conception of set?", in Logic, foundations of mathematics and computability theory, edited by R. Butts and J. Hintikka, Dordrecht, Holland, D. Reidel, 1977, 335-367.

[41] Plato, The Republic of Plato, translated by Francis Cornford (Oxford, Oxford University Press, 1945)

[42] Proclus, Commentary on Euclid's elements I, translated by G. Mor- 
row, (Princeton, Princeton University Press, 1970).

[43] Putnam, Hilary, "Mathematics without foundations", Journal of Philosophy 64 (1967): 5-22.

[44] Rayo, Agustín, "Beyond plurals", in Absolute generality, edited by Agustín Rayo and Gabriel Uzquiano (Oxford, Oxford University Press, 2006): 220-254.

[45] Robinson, Abraham, "Formalism 64", in Proceedings of the 1964 International Congress for Logic, Methodology, and Philosophy of Science, edited by Yehoshua Bar-Hillel, Amsterdam, North Holland, 1965, 228-246.

[46] Rumfitt, Ian, The boundary stones of thought: an essay in the philosophy of logic, Oxford, Oxford University Press, 2-15.

[47] Schaffer, Jonathan, "Monism: the priority of the whole", Philosophical Review 119 (2010): 31-76.

[48] Shapiro, Stewart, Foundations without foundationalism: a case for second-order logic (Oxford, Oxford University Press, 1991).

[49] Shapiro, Stewart, Thinking about mathematics: the philosophy of mathematics (Oxford, Oxford University Press, 2000).

[50] Simpson, Alex K., The proof theory and semantics of intuitionistic modal logic, Ph.D. Dissertation, University of Edinburgh, 1994.

[51] Sorabji, Richard, Time, creation and the continuum: theories in antiquity and the early middle ages (Chicago, University of Chicago Press, 2006).

[52] Troelstra, A. S., "Realizability", in Handbook of proof theory: Studies in logic and the foundations of mathematics 137, edited by Samuel R. Buss, Amsterdam, North Holland Publishing Company, 1998, pp. 407-473.

[53] Uzquiano, G. "Plural Quantification and Modality", Proceedings of the Aristotelian Society 111 (2011): 219-250.

[54] Weyl, Hermann, "Über die neue Grundlagenkrise der Mathematik", Mathematische Zeitschrift 10 (1921): 39-79.

[55] White, Michael J., The continuous and the discrete: ancient physical theories from a contemporary perspective (Oxford, Oxford University Press, 1992).

[56] Williamson, Timothy, Modal logic as metaphysics (Oxford, Oxford University Press, 2013). 